\title{
A Rapid Screening Measure for the Identification of Visuospatial Learning Disability in Schools
}

Cesare Cornoldi, Annalena Venneri, Fabio Marconato, Adriana Molin, and Cinzia Montinari

\begin{abstract}
Verbal and nonverbal forms of developmental learning disabilities have been reported. Whereas there are several instruments for evaluating children for the presence of verbal learning disabilities at school, no screening tool is available to identify children who have nonverbal learning disabilities. This study aimed at devising and validating a short screening questionnaire that can be used by teachers in primary schools to identify those children who need to be referred for clinical services because they have a visuospatial learning disability (VSLD). An 18-item Shortened Visuospatial Questionnaire (SVS) was derived from an earlier, longer version. Its validation procedure was twofold and was achieved by (a) verifying that children who had been identified with the SVS questionnaire as having a VSLD actually showed visuospatial deficits on psychometric evaluation and by (b) rating with the SVS a clinically identified population of children with VSLD. The results of the validation procedure showed that the SVS is a quick, reliable, and valid instrument that may be helpful in identifying children with VSLD in primary school.
\end{abstract}

A large body of evidence (see Wong, 1996, for an overview) has shown that there are subgroups within the large, ill-defined population of individuals with learning disabilities (LD). One large subgroup includes individuals whose underlying neuropsychological deficits include linguistic abilities-sometimes purely verbal, sometimes associated with visual deficits (Eden, Stein, Wood, \& Wood, 1995). There is, however, a small subgroup with intact verbal abilities but with deficits in areas of cognition other than language-in particular, visuospatial and visuomotor areas (Brumback, Harper, \& Weinberg, 1996; Fisher, DeLuca, \& Rourke, 1997; Rourke, 1982; Voeller, 1986).

This latter group has been studied in different ways, with emphasis on different definition criteria depending on the specific theme under study. In particular, Weintraub and Mesulam (1983) reported on a group of 14 patients, mainly young adults, who had a pat- tern of cognitive and emotional impairment that the authors associated with dysfunction of the right hemisphere. A few years later, Tranel, Hall, Olson, and Tranel (1987) described a group of 11 patients with striking neuropsychological evidence of right hemisphere dysfunction, including severe deficits in nonverbal intelligence, visual memory, and visuospatial functions. Neurological and functional evidence of right hemispheric dysfunction was collected by Nichelli and Venneri (1995), who studied a 22-yearold male patient and found marked hypometabolism of the right hemisphere using positron emission tomography (PET).

Groups of individuals with a pattern of cognitive and learning disabilities similar to the one involved in cases of right hemispheric dysfunction have been described by Rourke and coworkers in a series of studies (Rourke, 1982, 1987, 1993; Rourke \& Conway, 1997; Strang \& Rourke, 1983). The pattern of impairment shown by these individuals was described as nonverbal syndrome, or nonverbal learning disability. Children with nonverbal learning disabilities have clear signs of deficits typically associated with right hemisphere functioning but relatively wellpreserved mastery of linguistic abilities. These children present visuospatial difficulties, emotional difficulties, and learning problems in areas involved in visuospatial functioning, such as geometry, science, and so on. In a series of studies, Cornoldi et al. (1997) have focused their attention on a group with a similar type of visuospatial disability. This group was more specifically defined by the following three criteria:

1. learning disability confined to the learning of nonverbal material,

2. presence of a discrepancy between verbal and nonverbal/spatial IQ scores, and

3. failure in cognitive neuropsychological tests involving visuospatial 
memory but not affecting verbal memory.

The large variety of studies already available opens a window to an important subgroup of LD that lacks a clear definition and a reliable tool for early identification. Interestingly, most of the earlier studies have been case reports whose underlying deficit was identified in adult life (Nichelli \& Venneri, 1995; Tranel et al., 1987; Voeller, 1986; Weintraub \& Mesulam, 1983). Compared to learning problems affecting verbal abilities, which are usually identified very early in the school career, nonverbal difficulties are often brought to clinical attention at a much later point. As a consequence, intervention often starts too late, in a phase of development when rehabilitation may be less effective.

In this article, we will study this population with special reference to those children who manifest clear evidence of visuospatial disorders and meet the identification criteria suggested by Cornoldi et al. (1997). The identification of a population of children with a specific visuospatial disorder in the absence of verbal deficits should improve the understanding of their learning difficulties and of their neuropsychological problems. The study of a more select sample avoids the confounds induced by the study of a clinical population of children who have a more generalized learning problem that extends also to linguistic aspects of cognition. We will adopt the term visuospatial learning disability (VSLD) to refer to these children. This term emphasizes the visuospatial specificity of the disorder (which received less attention in the use of the term nonverbal learning disability). Furthermore, it avoids anatomical labeling. In fact, the identification criteria set by Cornoldi et al. (1997) do not take into account, and do not require, evidence from neurological or neuroimaging examinations.

It must be noted that several procedures are available for the psychological evaluation of visuospatial abilities, such as the Developmental Test of Visual Motor Integration (VMI; Beery, 1989) and the Developmental Test of Visual Perception (Hammill, Pearson, \& Voress, 1994), but no procedures are available for the detection of learning difficulties associated with visuospatial deficits in primary school. Whereas several basic procedures are available to be used by teachers in school to identify and monitor other forms of learning difficulties (e.g., reading and writing achievement tests, simple teacher rating scales such as the Teacher Rating Scale by Conners, 1990, used for detecting attention problems), no simple, preliminary procedures are available to be used by teachers to identify VSLD in school and to provide basic information about a child, which can then be passed on when the child is referred to a specialist.

To overcome this problem, Cornoldi et al. (1997) devised a visuospatial questionnaire (VS) to be used by teachers, including 37 items geared to the evaluation of specific features that characterize the deficit profile of children with nonverbal learning difficulties. Over time, however, it became apparent that this questionnaire had several shortcomings. It has proved to be too long to administer; some of its items did not have high discriminatory power; and it was not easily adaptable outside the Italian school system. To overcome these problems, we have devised a new questionnaire, which has the advantages of being easy to use and adaptable to cross-cultural use.

The questionnaire, which has been named Shortened Visuospatial Questionnaire (SVS), includes 18 items. Teachers are asked to evaluate whether a child presents a given characteristic on a 4-point scale (see Appendix for a full English version of the questionnaire). Ten items cover some of the deficits that according to previous studies represent the core critical features of VSLD. The following features are evaluated: use of the available space on paper while drawing, visuomotor coordination, comprehension of visuospatial relations on verbal description, coordination of complex movements, handling of the spatial components of calculation, spatial orientation, drawing, visuospatial learning, skills in observing the surrounding environment, and ability to deal with novel objects. These items are used to obtain a basic VSLD score. The questionnaire also includes two items that relate to the evaluation of other aspects typically associated with VSLD, but these scores are not included in the total score. They concern the handling of interpersonal skills (Item 9) and mathematical learning (Item 11). Two items (Items 13 and 14) collect preliminary information evaluating the presence of comorbidity with attention-deficit/hyperactivity disorder (ADHD; see Sandson, Bachna, \& Morin, 2000). Finally, four more items are included as controls. They collect information on a child's verbal abilities (Items 1 and 10) and a teacher's estimate of the child's overall cognitive potential (Item 17) and sociocultural level in general (Item 18). These items were selected as a result of a series of pilot studies where we found positive indications of their use. As a result of these pilot studies, the wording of some of the items had to be simplified. The reworded items were more acceptable to teachers who, in this context, preferred more general and simple formulations (e.g., "Does the child have good spatial orientation abilities?") rather than detailed formulations (e.g., including a specification of the behaviors and of the situations in which children could manifest their spatial orientation ability). We also examined the interrater reliability of the ratings given to 60 children by three independent teachers. The Pearson correlations for the overall VSLD scores were very high, with an interrater reliability ranging between .90 and .95 . This information was the basis that preceded the large-scale study described here.

In the cross-cultural study reported here, we have collected information on the utility, reliability, and validity of the SVS questionnaire. In the first study, 4,026 Italian children were evaluated by 200 primary school teachers. 
This procedure allowed us to collect information on the total distribution of scores on the SVS questionnaire in the general population of children and to identify a group of 54 children whose profile was indicative of VSLD. These children were then compared with a control group on a series of variables, to confirm the validity of the SVS questionnaire as a screening tool for visuospatial learning disability. In the second study, a different approach to validation was taken. Groups of British children clinically identified as having either dyslexia or VSLD were contrasted with a control group, and their scores on the SVS were compared.

\section{STUDY 1}

\section{Phase 1}

\section{Method}

Participants. A total of 4,026 (51\% boys, $49 \%$ girls) children were rated by their teachers with the SVS questionnaire. Thirty percent of children were in Grade 3, 30\% in Grade 4, 26\% in Grade 5, and 14\% in Grade 6, with an age range between 8 and 13 years. These children were recruited from schools in northeastern Italy, and they were selected to be representative of the child population in that area. Northeastern Italy is characterized by a majority of people living in urban contexts (midsize towns), and only a minority of people (about 10\%) lives in rural areas. The main occupational activities in this area are agriculture (about 7\%), industry and construction $(21 \%)$, clerical work $(7.5 \%)$, commerce (9\%), freelance professionals (5\%), and craftsmanship (5\%; Unioncamere, 2000).

Procedure. Teachers were nominated by the schools that were contacted with the collaboration of the Regional Center for Educational Research (IRRSAE) of Mestre/Venice. They were invited to collaborate in this study, and only those willing to take part received a copy of the SVS questionnaire and the associated instructions. The design of the SVS questionnaire required that the teachers familiarize themselves with the questionnaire beforehand and then proceed with a period of observation during classes. Once this preliminary work had been done, the actual completion of the questionnaire was rapid and took just a few minutes per child.

\section{Results}

Teachers reported that the instructions for the SVS questionnaire were easy to understand and that they did not find completing the questionnaire difficult. The VSLD score was computed on the basis of the 10 specific items. VSLD problems were associated with low scores. As expected, the distribution was skewed toward the highest scores, because a high proportion of children $(39 \%)$ did not have any particular VSLD and achieved scores between 35 and the maximum score of 40 . Another large group scored in the range between 29 and 34. The mean VSLD score achieved by the whole sample was 31.09 (SD = 7.10), with a slight but not significant variation between grades. There was a slight difference in score between girls $(M=31.91)$ and boys ( $M=30.31)$, but it was not significant.

Based on the distribution, a basic cutoff was computed, and this was associated with the tenth percentile (corresponding to a score of 21, approximately 1.5 SD below the mean). A more conservative cutoff was also identified, and this corresponded to a score of 17 (about -2 SD), involving $3.8 \%$ of the tested population. To identify a group with a highly specific, although not necessarily severe, VSLD profile, children had to meet the following additional criteria:

1. VSLD score below the basic cutoff (less than 10th percentile);

2. verbal score, as defined by the sum of scores on Items 1 and 10, above the 30th percentile $(\geq 5)$;

3. exclusion of poor overall cognitive abilities (score $>1$ on Item 17);
4. exclusion of very poor socioeconomic status (based on the response on Item 18).

It should be noted that only 54 out of a potential higher number of candidates met these criteria, as many children with a low VSLD score also had a low verbal score (the correlation, in the overall sample, between the two scores was very high, Pearson's $r=.80$ ). From the same sample we extracted a group of potential control children meeting Criteria 2, 3, and 4, whose VSLD score was above the 50th percentile, corresponding to a score $>31 ; 1,741$ children met these criteria. Within the latter group we grouped the third and fifth graders $(n=1,053)$ and contrasted their scores with those of the VSLD group on the other items of the SVS questionnaire. The results of the two groups were significantly different for both associated items. On Item 9, evaluating interpersonal skills, the mean score of the VSLD group was 2.39, whereas the mean score of the control group was $3.46, t(1105)=7.68, p<.01$, with a $d$ measure of effect size $=1.06$. On Item 11, evaluating mathematical learning, the mean scores were 2.41 and 3.54 for the VSLD and control groups, respectively, $t(1105)=9.41, p<.01, d=$ 1.30. Note that on this latter item, there were significant differences between grades, supporting our decision that this item should not be included in the VSLD score. The differences between groups on the other items of the SVS were not significant.

After 1 year, it was possible to obtain new ratings by the same teachers for 208 children from the original large sample. Despite the fact that the teachers did not remember their previous evaluations and that there might have been changes in the children's profile over such a long period, the teachers gave fairly similar ratings. The mean rating for the group at the first evaluation $(M=30.74, S D=7.11)$ was only slightly lower than that of the second evaluation $(M=31.18, S D=6.78)$. The correlation between the two ratings was acceptable (Pearson's $r=.76$ ). 


\section{Phase 2}

Validation was achieved by testing two small groups of children, including 18 VSLD group children and 18 controls, selected from the larger groups described earlier, whose families gave consent for extensive individual testing. The battery used for extensive testing included tasks specifically related to the VSLD problem, a few control tasks, and one task (visual span for verbalizable pictures) meant to assess the effect of verbal coding on a visual span task.

\section{Method}

Participants. The VSLD group included 12 boys and 6 girls, mean age 10.10. The control sample included children who had been randomly selected from the group of children who could be matched to the VSLD children for gender, age, grade, and teacher.

Tasks. Children were tested with the following tasks:

Verbal free recall. Fourteen unrelated concrete, high-frequency words were presented aurally at a rate of one word per second. The child had to orally recall these words immediately after the presentation of the entire list.

Span tasks. The span tasks were administered following the conventional procedure for span tasks-that is, a gradual increase in the number of items for the sequence, if the preceding sequence had been correctly reproduced. In case of error, another sequence of the same length as the initial sequence whose reproduction had been incorrect was presented. In case of error in two consecutive sequences of the same length, the administration of the task was stopped and the child's span was defined as the longest sequence correctly reproduced. The starting sequence length for the forward digit span task and the forward visuospatial span task was three items. In all other span tasks, the initial sequence length was two items.
Digit span forward and backward was measured using Wechsler's (1986) procedure and was defined by the longest sequence that the child could repeat correctly in serial order at least once.

Visuospatial span forward and backward was measured using the Corsi Block Test (Milner, 1971). The children were presented with a sequence of blocks, and they had to repeat the sequence in either the same or reverse order.

The house visual span (without verbal cues) task included a series of visually different houses. The items of each series were presented simultaneously. As the verbal label for each item was the same, children could not rely on any verbal support to perform this task. The task used a span-type procedure, but visual recognition of the items was required because the target items were presented together with an equal number of distractors.

The visual span for verbalizable pictures (figure span) task was identical to the previous one, except that the items were all different and could be easily verbally recoded (e.g., bear, jacket).

School achievement measures. Two tests were used as a measure of verbal and arithmetical learning. One was a reading achievement task, and the other consisted of a series of calculation tasks.

Verbal and visuospatial measures. The two subtests from the Wechsler Intelligence Scale for Children-Revised (WISC-R; Wechsler, 1986) scale that are most likely to tap into pure verbal or visuospatial abilities were selected. These were the Vocabulary subtest and the Block Design subtest, respectively.

\section{Results}

The mean scores of the two groups were compared using the Student $t$ test, with a Bonferroni correction that took into account the number of multiple comparisons. Table 1 presents the mean scores of the two groups and a measure of the effect size. The two groups had similar scores on the verbal free recall task, $p>.5$, with the mean scores of the two groups being largely within the 95\% confidence intervals (CI; 5.22-6.22). Significant differences between the groups (with mean scores largely beyond the $95 \%$ confidence intervals) were present on the scores on

\begin{tabular}{|c|c|c|c|c|c|}
\hline \multicolumn{6}{|c|}{$\begin{array}{c}\text { TABLE } 1 \\
\text { Scores on Psychometric Tasks Achieved by the VSLD Group Identified by } \\
\text { the SVS and Their Matched Controls }\end{array}$} \\
\hline \multirow[b]{2}{*}{ Task } & \multicolumn{2}{|c|}{ VSLD group } & \multicolumn{2}{|c|}{ Control group } & \multirow[b]{2}{*}{ Effect size $(d)$} \\
\hline & $M$ & $S D$ & $M$ & $S D$ & \\
\hline \multicolumn{6}{|l|}{ WISC-R } \\
\hline Vocabulary & 11.68 & 2.86 & 11.35 & 2.59 & .12 \\
\hline Block design & 11.50 & 2.92 & 16.17 & 2.37 & $-1.79^{\star *}$ \\
\hline \multicolumn{6}{|l|}{ Reading } \\
\hline Accuracy & 4.20 & 2.90 & 3.56 & 2.72 & .23 \\
\hline Speed & 0.32 & 0.13 & 0.30 & 0.09 & .18 \\
\hline \multicolumn{6}{|l|}{ Memory } \\
\hline Digit span & 8.56 & .98 & 9.89 & 1.49 & $-1.03^{\star *}$ \\
\hline Verbal free recall & 5.33 & 1.41 & 6.11 & 1.49 & .54 \\
\hline House span & 2.73 & .67 & 3.31 & .52 & $-.98^{\star *}$ \\
\hline Figure span & 5.01 & .95 & 6.11 & 1.43 & $-.89^{\star}$ \\
\hline Corsi block span & 8.78 & 1.17 & 10.78 & 1.11 & $-1.76^{\star \star}$ \\
\hline \multicolumn{6}{|l|}{ Calculation } \\
\hline Accuracy & 21.01 & 2.25 & 23.83 & 2.25 & $-1.25^{*}$ \\
\hline Speed & 547.40 & 220.10 & 467.74 & 110.30 & $.45^{*}$ \\
\hline
\end{tabular}

Note. VSLD = visuospatial learning disability; WISC-R = Wechsler Intelligence Scale for Children-Revised (Wechsler, 1986); SVS = Shortened Visuospatial Questionnaire. ${ }^{*} p<.05 .{ }^{* *} p<.01$. 
the visuospatial span, $t(34)=3.95, p<$ $.01, \mathrm{CI}=9.27-10.29$; the visual span without verbal cues (house span), $t(34)=2.91, p<.01, \mathrm{CI}=2.80-3.24$; the visual span for verbalizable pictures (figure span), $t(34)=2.71, p<.05, \mathrm{CI}=$ 5.11-6.00; and the digit span, $t(34)=$ 3.17, $p<.01, \mathrm{CI}=8.74-9.70$.

Raw scores on the two WISC-R subtests were transformed into corrected scores using the Italian adaptation manual (Wechsler, 1986). Analyses showed no significant difference in the Vocabulary subtest, $t(34)=0.35$, ns, whereas a large difference was present in the Block Design subtest, $t(34)=$ $5.17, p<.01$. The relationship between a specific visuospatial intelligence score and the VSLD score obtained with the SVS questionnaire was further confirmed by the patterns of Pearson's correlation, as the VSLD correlated significantly with the Block Design score, $r=.65, p<.001$, but not with the Vocabulary score, $r=-.005$.

No significant difference between the two groups was found on the reading achievement task, neither for accuracy of reading, $p=.52$, nor for speed, $p=.71$. A significant difference between the two groups was observed in the arithmetic test for both accuracy of performance, $p=.015$, and speed, $p=$ .013 (see Table 1).

\section{Discussion}

The questionnaire was easy to complete, and the teachers did not report any difficulties in using it to evaluate the children's performance. Despite its simplicity, the questionnaire demonstrated high sensitivity and specificity and flagged those children who met the three diagnostic criteria set by Cornoldi et al. (1997) to be referred for further investigation for the presence of VSLD. The children identified as having VSLD presented scores on a verbal intelligence subtest comparable to those of the control group, whereas their scores on the nonverbal subtest showed the expected lower performance than the controls. Full IQ testing was not permitted for these children, and only these two subtests could be used. The scores on these subtests, however, seem to be the ones that most accurately reflect verbal and nonverbal abilities among the subtests included in the Wechsler scales.

The VSLD group children showed good linguistic learning, as demonstrated by their high performance on the reading achievement task, whereas they showed learning difficulties in the arithmetical tasks, confirming the findings of previous research (e.g., Rourke, 1993). These difficulties are highlighted both by the teachers' ratings on those items evaluating mathematical learning and by the children's specific performance on the calculation test.

The extensive memory evaluation of the children revealed that the VSLD group had a specific difficulty with visuospatial memory tasks, as suggested by previous research (e.g., Cornoldi, Rigoni, Tressoldi, \& Vio, 1999). This difficulty involved the performance not only on spatial and visual span tasks but also on a visual memory task with verbalizable items, suggesting that this task is largely supported by visual cues and that the difficulties in coding these cues can affect the overall performance. As for the two mainly verbal memory tasks, the performance of the VSLD group did not differ from that of the controls in the semantic free recall task, but a significant difference was observed in the scores on the digit span task. This difference could be ascribed to the fact that this latter task is less strictly linguistic, and therefore less lateralized, and loads on working memory resources to a greater extent.

It is worth noting that in order to obtain a highly selective VSLD group free from any potential confound, only a subgroup of the larger group having a very low SVS score was selected. However, no child in this VSLD group had a performance falling above the mean on the visuospatial memory tasks administered. Therefore, no child could be considered a false positive. Similarly, no control child presented a generalized pattern of difficulty in the tasks and could, therefore, be consid- ered a false negative. Finally, we observed that the VSLD group achieved a significantly lower rating on the item evaluating interpersonal skills, confirming the previously reported observation of an association of this disorder with interpersonal difficulties.

\section{STUDY 2}

Two main points remained open, namely (a) cross-validating the questionnaire to make sure that it could be readily adapted to a different school system, and (b) evaluating whether the questionnaire was able to discriminate a clinically identified population of children with VSLD from typical children and discriminate the VSLD sample from other LD samples (e.g., children with dyslexia).

\section{Method}

Three groups of children were selected. Group 1 included 10 children with VSLD ( 9 boys and 1 girl) between 6 and 12 years old. These children were recruited from referrals to the Occupational Therapy Unit at the Royal Children's Hospital and from a special unit for children with learning difficulties in a primary school in Aberdeen, UK. Five of these children were participating in a rehabilitation program for their visuomotor difficulties. All children had been identified as having VSLD by an educational psychologist and undergone a full psychometric evaluation. In particular, all children had learning difficulties, visuospatial neuropsychological problems, and a WISC-R Verbal IQ > Performance IQ discrepancy larger than 20 points, but their Full Scale IQ fell in the average range.

Group 2 included 10 children with dyslexia ( 7 boys and 3 girls) between 9 and 11 years old. All children with dyslexia were recruited from a special dyslexia unit in a primary school in Aberdeen, UK. Their reading abilities were evaluated using the Wide Range 
Achievement Test (Jastak, 1978). All children had previously been evaluated by an educational psychologist, and their Full Scale IQ fell in the average range. The third group (control group) included 50 children between 5 and 12 years old randomly selected from the same school as the dyslexia group and with a sociocultural background to match both clinical groups.

The SVS questionnaire was completed by the support teachers for the two LD groups and by the classroom teachers for the control group.

\section{Results}

To have indicative data from a large, randomly selected control group, the proportion of boys and girls in this group was roughly equal. Therefore, the differences between this group and the two experimental groups could be due to the different proportions of boys and girls represented. To rule out this possibility, we examined the effect of gender in the control group on the scores in the critical variables. A series of $t$ tests showed no significant gender difference in the VSLD score $(p=.84)$, the linguistic score $(p=.20)$, or the scores on Item $9(p=.58)$ and Item 11 $(p=.23)$.

A series of analyses of variance contrasted the three groups on the separate relevant ratings of the questionnaire. First of all, an ANOVA demonstrated that the ratings on the aggre- gate scores for the specific items of the VSLD group were significantly lower than for the other groups, $F(2,67)=$ 10.17, $M S E=28.21, p<.01$. Post hoc paired comparisons using the Scheffé test showed significant differences between the VSLD group and both the dyslexia group, $p<.001$, and the control group, $p<.001$, whereas the comparison between the latter two groups was not significant, $p=.50$. Inspection of individual VSLD scores showed that only one false negative was present in the VSLD group. Within the other groups, no child had a particularly low VSLD rating, except for two children in the control group whose scores were borderline.

A further ANOVA was performed on the scores on the items for associated symptoms. On Item 9 (social interactions), the expected significant difference between groups was observed, $F(2,67)=8.16, M S E=.61, p<.001$. Post hoc paired comparisons using the Scheffé test showed significant differences between the VSLD group and both the dyslexia group, $p<.001$, and the control group, $p<.01$, whereas the comparison between the latter two was not significant, $p=.11$. A similar pattern was observed for Item 11 (math learning). There was a significant difference between the three groups, with the VSLD group achieving the lowest scores of all, $F(2,67)=22.78, M S E=.52$, $p<.01$. Post hoc paired comparisons using the Scheffé test showed significant differences between the VSLD group and both the dyslexia group, $p<$ .01 , and the control group, $p<.02$. A significant difference was also present in the comparison between the control group and the dyslexia group, with the latter group achieving the highest scores of all, $p<.01$.

When aggregate ratings on the control verbal items (Items 1 and 10) were compared, we found a significant difference between groups, $F(2,67)=8.21$, $M S E=2.41, p<.01$. The ratings achieved by the dyslexia group were lower than those of the other two groups; post hoc paired comparisons using the Scheffé test confirmed significant differences between the dyslexia group and both the VSLD group, $p<$ .01 , and the control group, $p<.001$, whereas the comparison between the latter two groups was not significant, $p=.99$.

A similar analysis was run for the aggregate ratings for the ADHD items (Items 13 and 14). There were no significant differences among the scores of the three groups, $F(2,67)=0.09$, $M S E=2.70, n s)$.

Table 2 presents the mean ratings of the three groups for the VSLD score, the two verbal items together, the two ADHD scores, and the associated items.

\section{GENERAL DISCUSSION}

Within the field of developmental learning disabilities, it is widely acknowl-

TABLE 2

Scores on the SVS Achieved by the Clinically Identified VSLD Group, the Dyslexia Group, and the Control Group

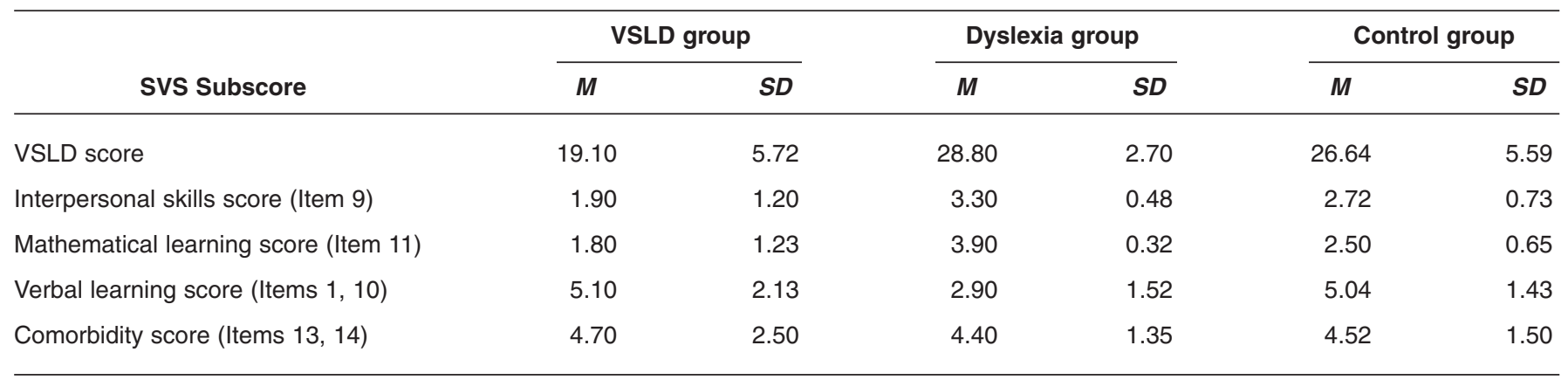


edged that forms other than linguistic LD can be found (e.g., Tranel et al., 1987; Voeller, 1986; Weintraub \& Mesulam, 1983). Despite this consensus and the availability of group studies of these forms of nonverbal learning disabilities, their characteristics, identification criteria, and assessment procedures are not unified and are poorly delineated. This situation has resulted in a variety of labels being used to refer to this population. Furthermore, different, not completely overlapping groups have been identified.

In contrast with the large and wellestablished pool of evaluation procedures available for linguistic LD that usually lead to the early and reliable detection of affected children, no straightforward instrument is available to teachers for a preliminary identification of nonverbal learning difficulties in the classroom. In this study, we attempted to offer an easy tool that may serve as a quick and valid screening procedure. In the educational system, there has been a biased emphasis toward the acquisition of literacy and its related problems. The SVS questionnaire proposed here should fill this gap and help teachers to identify children who, despite typical literacy acquisition, might need further clinical consideration and should eventually be brought to the attention of educational psychology or rehabilitation services.

Our studies have demonstrated that the SVS questionnaire can reliably help to identify children who, on deeper examination, show consistently lower achievement compared to controls on tests measuring visuospatial abilities. The SVS questionnaire is based on teacher ratings, which have been successfully used in the field of developmental disabilities for other purposes and problems (e.g., Conners, 1973). Obviously, a simple rating instrument used by teachers without specific training has some clear limitations, and its results should be interpreted cautiously. However, this twofold validation procedure demonstrated that with the use of an appropriate cutoff, the risks of false positives and false negatives are low. One strong point in the approach to validation in this study is the confirmation by psychometric testing that children rated by the SVS questionnaire as having VSLD did indeed show a visuospatial deficit when individually tested for these abilities. On the other hand, the SVS questionnaire was also able to discriminate those children who had been identified as having VSLD with other means by independent assessment, differentiating them not only from a control group but also from children with other forms of LD.

\section{ABOUT THE AUTHORS}

Cesare Cornoldi is professor of psychology in the Department of General Psychology at Padova University, Italy. His current interests include human learning, memory, spatial abilities, mental imagery, and learning disabilities. Annalena Venneri is senior lecturer in neuropsychology in the Cognitive Neuroimaging Research Unit, Department of Psychology and Functional Imaging Centre, University of $A b$ erdeen, UK. Her research interests focus on memory and language deterioration in dementia and on the cognitive characteristics underlying developmental deficits such as dyslexia and visuospatial learning disability. Fabio Marconato is a doctoral student in the Department of General Psychology at Padova University. His interests include cerebral lateralization, learning disabilities, and human and animal learning. Adriana Molin is a teacher and psychologist, with a research position at IRRE Veneto, Mestre. Her interests involve nonspecific learning disabilities and improvement of scholastic abilities such as attention, reading, and mathematical abilities. Cinzia Montinari is a professional psychologist involved in the field of cognitive and neuropsychological disabilities. Address: Cesare Cornoldi, Department of General Psychology, University of Padova, Via Venezia 8, 35131 Padova, Italy.

\section{AUTHORS' NOTES}

1. This study was supported by a grant from The Wellcome Trust to Annalena Venneri and Cesare Cornoldi.

2. The authors thank Margherita Garuti, Department of Psychology, University of Padova, and Elizabeth Stevenson, Depart- ment of Occupational Therapy, Royal Aberdeen Children's Hospital, for their help in children's recruitment and testing, and all teachers in Italy and UK who took part in this study.

\section{REFERENCES}

Beery, K. E. (1989). Developmental test of visual motor integration. Cleveland: Modern Curriculum Press.

Brumback, R., Harper, C., \& Weinberg, W. (1996). Nonverbal learning disabilities, Asperger's syndrome, pervasive developmental disorder-Should we care? Journal of Child Neurology, 2, 427-429.

Conners, C. K. (1990). The Connors rating scales. North Tonawanda, NY: MultiHealth Systems.

Cornoldi, C., Friso, G., Giordano, L., Molin, A., Poli, S., Rigoni, F., et al. (1997). Abilita' visuospaziali [Visuospatial abilities]. Trento, Italy: Erickson.

Cornoldi, C., Rigoni, F., Tressoldi, P. E., \& Vio, C. (1999). Imagery deficits in nonverbal learning disabilities. Journal of Learning Disabilities, 32, 48-57.

Eden, G. F., Stein, J. F., Wood, M. H., \& Wood, F. B. (1995). Verbal and visual problems in reading disability. Journal of Learning Disabilities, 28, 272-290.

Fisher, N., DeLuca, J., \& Rourke, B. (1997). Wisconsin Card Sorting Test and Halstead category test performances of children and adolescents who exhibit the syndrome of nonverbal learning disabilities. Child Neuropsychology, 3(1), 61-70.

Hammill, D., Pearson, N. A., \& Voress, J. K. (1994). Developmental test of visual perception. Austin, TX: PRO-ED.

Jastak, S. (1978). Wide range achievement test. Wilmington, DE: Jastak.

Milner, B. (1971). Interhemispheric differences in the localization of psychological processes in man. British Medical Bulletin, 27, 272-277.

Nichelli, P., \& Venneri, A. (1995). Right hemisphere developmental learning disability: A case study. Neurocase, 1, 173177.

Rourke, B. (1982). Central processing deficiencies in children: Toward a developmental neuropsychological model. Journal of Clinical Neuropsychology, 4(11), 1-18.

Rourke, B. (1987). Syndrome of nonverbal learning disabilities: The final common pathway of white-matter disease/ dysfunction? The Clinical Neuropsychologist, 1, 209-234. 
Rourke, B. (1993). Arithmetic disabilities, specific and otherwise: A neuropsychological perspective. Journal of Learning Disabilities, 26, 214-226.

Rourke, B., \& Conway, J. (1997). Disabilities of arithmetic and mathematical reasoning: Perspectives from neurology and neuropsychology. Journal of Learning Disabilities, 30, 34-36.

Sandson, T. A., Bachna, K. J., \& Morin, M. D. (2000). Right hemisphere dysfunctioning in ADHD. Journal of Learning Disabilities, 33, 83-90.
Strang, J., \& Rourke, B. (1983). Conceptformation/nonverbal reasoning abilities in children who exhibit specific academic problems with arithmetic. Journal of Clinical Child Psychology, 12(1), 33-39.

Tranel, D., Hall, L., Olson, S., \& Tranel, N. (1987). Evidence for a right-hemisphere developmental learning disability. Developmental Neuropsychology, 3, 113-127.

Unioncamere. (2000). Relazione sulla situazione economica del Veneto [Report on Veneto economical situation]. Venice, Italy: Ufficio Studi Unioncamere.
Voeller, K. (1986). Right hemisphere deficit syndrome in children. American Journal of Psychiatry, 143, 1004-1009.

Wechsler, D. (1986). Scala di intelligenza per bambini riveduta [Intelligence scale for children-Revised]. Firenze, Italy: Organizzazioni Speciali.

Weintraub, S., \& Mesulam, M. (1983). Developmental learning disabilities of the right hemisphere: Emotional, interpersonal, and cognitive components. Archives of Neurology, 40, 463-468.

Wong, B., Y., L. (1996). The ABCs of learning disabilities. San Diego: Academic Press.

\section{APPENDIX}

Shortened Visuospatial Questionnaire. For each item, the mean ratings obtained by the original sample, and the associated standard deviations, are reported in parentheses. Items preceded by VSLD are used for obtaining the VSLD score.

\begin{tabular}{|c|c|c|c|c|}
\hline Questions & 1 & 2 & 3 & 4 \\
\hline \multicolumn{5}{|l|}{ 1. Can the child easily memorize material such as names, information, and poems? $(2.96,1.00)$} \\
\hline \multicolumn{5}{|l|}{ VSLD 2. Is the child able to make use of the available space when drawing? $(3.09, .95)$} \\
\hline \multicolumn{5}{|l|}{$\begin{array}{l}\text { VSLD 3. Can the child use tools, such as scissors, set square, or ruler, that require } \\
\text { independent and coordinated use of both hands? }(3.14, .93)\end{array}$} \\
\hline \multicolumn{5}{|l|}{$\begin{array}{l}\text { VSLD 4. Does the child understand spoken commands or texts that involve space } \\
\text { relationships? }(3.08, .93)\end{array}$} \\
\hline \multicolumn{5}{|l|}{$\begin{array}{l}\text { VSLD } 5 \text {. Is the child able to execute complex everyday movements, such as tying } \\
\text { shoelaces? }(3.53, .79)\end{array}$} \\
\hline \multicolumn{5}{|l|}{$\begin{array}{l}\text { VSLD } 6 \text {. Does the child show good understanding of spatial relationships in calculation, and can } \\
\text { he or she write numbers in column correctly? }(3.19, .93)\end{array}$} \\
\hline \multicolumn{5}{|l|}{ VSLD 7. Does the child have good spatial orientation abilities? $(3.27, .87)$} \\
\hline \multicolumn{5}{|l|}{ VSLD 8 . Is the child good at drawing? $(2.96, .93)$} \\
\hline \multicolumn{5}{|l|}{ 9. Can the child easily interact with friends? $(3.10, .88)$} \\
\hline \multicolumn{5}{|l|}{ 10. Has the child reached a good linguistic learning level for his or her age? $(2.87, .97)$} \\
\hline \multicolumn{5}{|l|}{ 11. Has the child reached a good mathematical learning level for his or her age? $(2.90, .98)$} \\
\hline \multicolumn{5}{|l|}{ VSLD 12. Is the child competent in learning contexts that rely on visuospatial skills? $(3.00, .92)$} \\
\hline \multicolumn{5}{|l|}{ 13. Is the child distracted easily? $(2.22,1.01)$} \\
\hline \multicolumn{5}{|l|}{ 14. Is the child often restless or hyperactive? $(1.77, .99)$} \\
\hline \multicolumn{5}{|l|}{ VSLD 15. Is the child a good observer of the environment in which he or she lives? $(2.89, .96)$} \\
\hline \multicolumn{5}{|l|}{$\begin{array}{l}\text { VSLD 16. Does the child demonstrate an interest in new objects, and can he or she deal with } \\
\text { them? }(2.92, .94)\end{array}$} \\
\hline \multicolumn{5}{|l|}{ 17. Does the child show good overall cognitive potential? $(2.97, .96)$} \\
\hline 18. Does the child have a poor sociocultural background? $(1.46, .89)$ & & & & \\
\hline
\end{tabular}


Copyright $\odot 2003$ EBSCO Publishing 